\title{
A thermodinamically consistent derivation of a frictional-damage cohesive-zone model with different mode I and mode II fracture energies
}

\author{
R. Serpieri \\ Dipartimento di Ingegneria, Università degli Studi del Sannio, Piazza Roma, 21 - I. 82100, \\ Benevento, Italy. \\ E. Sacco \\ Università di Cassino e del Lazio Meridionale, Dipartimento di Ingegneria Civile e Meccanica, \\ Cassino(FR), Italy. \\ G. Alfano \\ Brunel University, School of Engineering and Design, Uxbridge, UB8 3PH, UK.
}

\begin{abstract}
The present paper deals with the derivation of an interface model characterized by macroscopic fracture energies which are different in modes I and II, the macroscopic fracture energy being the total energy dissipated per unit of fracture area.

It is first shown that thermo-dynamical consistency for a model governed by a single damage variable, combined with the choice of employing an equivalent relative displacement and of a linear softening in the stress-relative displacement law, leads to the coincidence of fracture energies in modes I and II. To retrieve the experimental evidence of a greater fracture energy in mode II, a micro-structured geometry is considered at the typical point of the interface where a Representative Interface Element (RIE) characterized by a periodic arrangement of distinct inclined planes is introduced. The interaction within each of these surfaces is governed by a coupled damage-friction law.

A sensitivity analysis of the correlation between micromechanical parameters and the numerically computed single-point microstructural response in mode II is reported. An assessment of the capability of the model in predicting different mixed mode fracture energies is carried out both at the single microstructural interface point level and with a structural example. For the latter a double cantilever beam with uneven bending moments has been analyzed and numerical results are compared with experimental data reported in the literature for
\end{abstract}


different values of mode mixity.

Keywords: cohesive-zone models; fracture energies; mixed-mode fracture; damage-friction coupling.

\section{Introduction}

Cohesive zone models combining interface damage, friction and unilateral contact find primary applications in the description of an extensive family of degradation behaviors, involving macroscopic and/or microscopic surface debonding, experimentally observed both in homogeneous and in heterogeneous materials.

Two well known experimental findings of the mechanics of interface decohesion in quasi-brittle materials are: 1) the existence of coupling between damage progression and hysteretic frictional behavior and 2) the existence of an experimentally measured fracture energy in pure mode-II decohesion that is often significantly higher than its pure mode-I counterpart.

Several interface models accounting for damage-friction coupling have been proposed in literature, e.g., Del Piero and Raous (2010) and references therein. For quasi-brittle materials, asymptotic expansions of the crack-tip fields accounting for Coulomb-type friction law have been reported by Karihaloo and Xiao (2008) and by Barpi and Valente (2010). Several models capture the evolution from adhesion to frictional regime exploiting different strategies, with a progressive or a sudden transition (Raous et al., 1999). Among these models, Alfano and Sacco (2006), Alfano et al. (2006) and, more recently, Sacco and Toti (2010) introduced a method to combine interface damage and friction in a cohesive zone model based on a simplified micromechanical formulation. The main idea developed in these references is to combine damage and friction by considering a representative area at a micromechanical scale, which is assumed to be additively decomposed into an undamaged and a fully damaged part; moreover, it is supposed that friction occurs only on the latter. Damage is assumed to evolve according to the formulation originally addressed in Alfano and Crisfield (2001), while the frictional behavior is governed by a Coulomb law. A similar approach has been developed in Ragueneau et al. (2000) to define the stress-strain relationship for continuum media.

In the present contribution, a study of the damage-friction interface model is accomplished within the framework of thermodynamics with internal variables with a specific investigation on the possibility to account for the presence of different mode-I and mode-II fracture energies.

Email address: roberto.serpieri@unisannio.it (R. Serpieri)

Preprint submitted to European Journal of Mechanics - A/Solids

June 17, 2014 
With the purpose of devising a model capable to suitably address different mode-I and mode-II fracture energies while preserving a consistent standard thermodynamic formulation, a strategy for enhancing the approach exploited in Alfano and Sacco (2006) and generalized by Sacco and Toti (2010) is investigated in the present contribution.

The solution exploited for retrieving a greater fracture energy in mode-II is based on the enhancement proposed by Serpieri and Alfano (2011), whereby the geometry of the interface is represented in the form of a periodic arrangement of distinct inclined planes, denominated Representative Interface Element (RIE). The interaction within each of these surfaces is governed by the combined damagefriction interface formulation proposed in Alfano and Sacco (2006). Although a simple bilinear damage law with equal mode-I and mode-II fracture energies and no dilatancy is introduced on each individual plane, a macroscopic dilatant and hysteretic behavior of the interface law is obtained with a significant difference among mode-I and mode-II fracture energies.

Numerical applications are reported together with some details on the algorithmic implementation. In particular, a sensitivity analysis of the correlation between micromechanical parameters and the mixed-mode behavior of the model is carried out at the constitutive level in terms of macroscopically observed fracture energies. Moreover, to assess the applicability of the model to a full-scale structural problem, the results of finite-element simulations of the mixed-mode delamination of a Double Cantilever Beam subjected to Uneven Bending Moments (DCB-UBM) are also illustrated. In particular, the numerical results, as predicted by the proposed model, are compared with the experimental ones determined by Sørensen and Jacobsen (2009).

In the following braces are used to denote vector arrays, treating them as column vectors by default, while square brackets are used for matrices.

\section{Assumptions on state laws and damage evolution}

A cohesive zone model with relative displacement variables $\mathbf{s}=\left\{s_{n}, s_{t},\right\}^{t}$ is considered. A right-handed global reference frame $(n, t)$ is introduced, $n$ and $t$ being the tangential and normal directions of the planar interface $\mathcal{S}$. The interface is endowed with adhesion that can experiment damage driven by both normal and tangential relative displacements. A pure mechanical formulation is considered assuming that temperature is not significant for the model and it is consequently ruled out from the set of state variables. Damage is addressed by introducing a single damage variable $\alpha$. Following Del Piero and Raous (2010) $\alpha$ is the unique internal variable so that the whole set of normal state variables is $\chi=\{\mathbf{s}, \alpha\}^{t}$ (Suquet, 1982). 


\subsection{State law}

The Helmholtz free energy function for the cohesive zone model under study, denoted as:

$$
\Psi=\Psi(\mathbf{s}, \alpha)
$$

is assumed to be a convex function of $\mathbf{s}$ and a non-convex function of $\alpha$. The $\Psi$-conjugated generalized stress variables are denoted as:

$$
\mathbf{X}=\frac{\partial \Psi}{\partial \boldsymbol{\chi}}=\{\boldsymbol{\sigma}, X\}^{t}=\left\{\frac{\partial \Psi}{\partial \mathbf{s}},-\frac{\partial \Psi}{\partial \alpha}\right\}^{t}
$$

where $\boldsymbol{\sigma}=\left\{\sigma_{n}, \sigma_{t}\right\}^{t}$ is the reversible stress and $X$ is the damage-associated driving force.

To set the ideas, it is initially assumed $s_{n} \geq 0$ and $s_{t} \geq 0$; in this case, two landmark points are introduced in the space of the state variables: the onset of damage in pure mode I and pure mode II, respectively denoted as $\chi^{(I 0)}$ and $\chi^{(I I 0)}$ whose associated relative displacements are $\mathbf{s}^{(I 0)}=\left\{s_{n}^{0}, 0,\right\}^{t}$ and $\mathbf{s}^{(I I 0)}=$ $\left\{0, s_{t}^{0}\right\}^{t}$. Two further landmark state points are denoted as $\chi^{(I f)}$ and $\chi^{(I I f)}$. The former is the state at which zero normal stress is attained in a monotonic path in pure mode I. The latter is the state at which zero tangential stress is attained in a monotonic path in pure mode II. The associated relative displacements are $\mathbf{s}^{(I f)}=\left\{s_{n}^{f}, 0,\right\}^{t}$ and $\mathbf{s}^{(I I f)}=\left\{0, s_{t}^{f}\right\}^{t}$. Finally, the four points above introduced are characterized by the state variables:

$$
\begin{array}{ll}
\chi^{(I 0)}=\left\{\mathbf{s}^{(I 0)}, \alpha^{(I 0)}\right\}^{t}, & \chi^{(I I 0)}=\left\{\mathbf{s}^{(I I 0)}, \alpha^{(I I 0)}\right\}^{t} \\
\chi^{(I f)}=\left\{\mathbf{s}^{(I f)}, \alpha^{(I f)}\right\}^{t}, & \chi^{(I I f)}=\left\{\mathbf{s}^{(I I f)}, \alpha^{(I I f)}\right\}^{t}
\end{array}
$$

The generalized stress coordinates corresponding to the first attainment of $\chi^{(I 0)}$ and $\chi^{(I f)}$ in a pure mode I monotonic path are:

$$
\mathbf{X}^{(I 0)}=\left\{\boldsymbol{\sigma}^{(I 0)}, X^{(I 0)}\right\}^{t}, \quad \mathbf{X}^{(I f)}=\left\{\boldsymbol{\sigma}^{(I f)}, X^{(I f)}\right\}^{t}
$$

Similarly, the generalized stress coordinates corresponding to the first attainment of $\chi^{(I I 0)}$ and $\chi^{(I I f)}$ in a pure mode II monotonic path are:

$$
\mathbf{X}^{(I I 0)}=\left\{\boldsymbol{\sigma}^{(I I 0)}, X^{(I I 0)}\right\}^{t}, \quad \mathbf{X}^{(I I f)}=\left\{\boldsymbol{\sigma}^{(I I f)}, X^{(I I f)}\right\}^{t}
$$

Further hypotheses at the basis of the interface model are introduced as follows. 
I Natural state - The interface stress at zero relative displacements, $\mathbf{s}=\mathbf{0}$, is assumed to be always zero. Such a point of the displacement plane is denoted as $\mathbf{O}$.

II Initial elastic behavior in a neighborhood of the origin - It is assumed that in the displacement plane within a finite neighborhood $\Delta_{\mathbf{O}}$ of the zero relative displacement damage does not evolve, so that the behavior of the interface is completely elastic. Consequently, setting the internal variable $\alpha$ equal to the initial value $\alpha^{(0)}$, any curve in the $\chi$ space, whose points are all such that $\mathbf{s} \in \Delta_{\mathbf{O}}$, is a path characterized by a constant $\alpha=\alpha^{(0)}$ and, moreover,

$$
\alpha^{(I 0)}=\alpha^{(I I 0)}=\alpha^{(0)}
$$

The boundary of $\Delta_{\mathbf{O}}$ curve in the s space connecting points $\chi^{(I 0)}$ and $\boldsymbol{\chi}^{(I I 0)}$ is denoted as $\boldsymbol{\Gamma}_{0}$ and contains points $\mathbf{s}^{(I 0)}$ and $\mathbf{s}^{(I I 0)}$.

III Region of complete decohesion - It is assumed that a region of the displacement plane $\Delta_{\text {sep }}$ exists in which the state of complete decohesion is attained. The separation region $\Delta_{\text {sep }}$ is characterized by a vanishing Helmholtz free energy. Any displacement point belonging to this region, $\mathbf{s} \in \Delta_{s e p}$, is such that:

$$
\Psi=0
$$

for any previous loading/displacement history.

The boundary of $\Delta_{\text {sep }}$ in the s space is denoted as $\boldsymbol{\Gamma}_{f}$ and connects points $\chi^{(I f)}$ and $\chi^{(I I f)}$. When $\mathbf{s}$ belongs to $\Delta_{\text {sep }}$, the damage variable $\alpha$ has reached a final value $\alpha^{(f)}$ that cannot be modified for any other loadingunloading history.

Two further curves are introduced in the s space, denoted as $\boldsymbol{\Gamma}_{I}$ and $\boldsymbol{\Gamma}_{I I}$, that, respectively, represent pure mode I and mode II increasing displacement paths. Figure 1 shows a schematic plot in the $\mathbf{s}$ plane with the above introduced displacement-like and stress-like state points.

IV Uncoupled elastic behavior in pure modes I and II - Elastic uncoupling is assumed between the stress-relative displacement responses in modes I and II. More specifically, it is assumed that in a pure mode I, along $\boldsymbol{\Gamma}_{I}$ (mode II, along $\boldsymbol{\Gamma}_{I I}$ ) the stress response consists only in normal (tangential) stresses. Physically, this hypothesis amounts to state that the interface is perfectly plane, without any form of interlocking. This condition is mathematically stated in a synthetic and general formula

$$
\frac{\partial^{2} \Psi}{\partial s_{n} \partial s_{t}}=0, \quad \mathbf{s} \in \boldsymbol{\Gamma}_{I}, \mathbf{s} \in \boldsymbol{\Gamma}_{I I}
$$




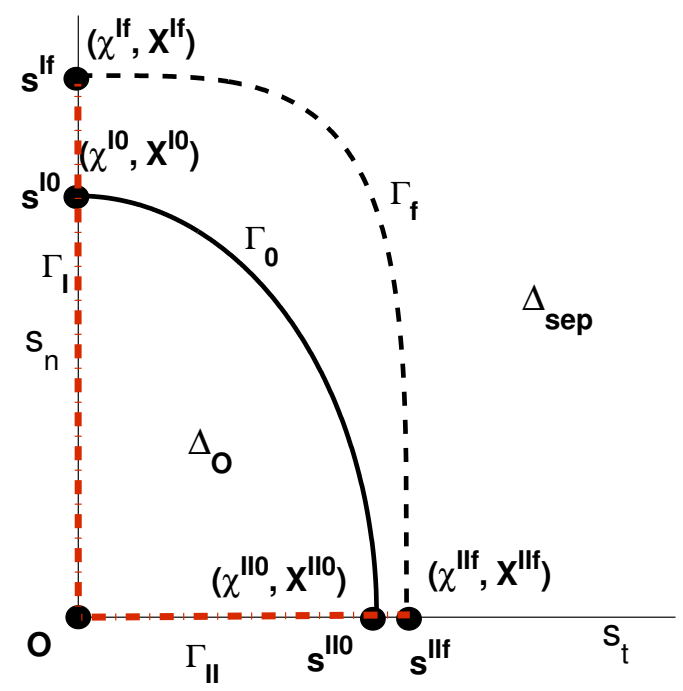

Figure 1: Schematic plot of landmark points, regions and curves in the s space.

and implies that when the interface is subjected to a monotonic relative displacement path along $\boldsymbol{\Gamma}_{I}$ the stress response contains no tangential stress and vice-versa, i.e. when the interface is subjected to a relative displacement path along $\boldsymbol{\Gamma}_{I I}$, the stress response exhibits no normal stress.

V Equivalent relative displacement in mixed mode - An equivalent relative-displacement scalar function $s^{e q}(\mathbf{s})$ can be introduced whose maximum attained value during the past loading history is indicated as $\bar{s}^{e q}$

$$
\bar{s}^{e q}(t)=\max _{\text {history }} s^{e q}(\mathbf{s})=\max _{\tau \in\left[t_{0}, t\right]} s^{e q}(\mathbf{s}(\tau))
$$

where $t_{0}$ is the initial time of the analysis, $t$ is the current time and it is assumed that the damage variable depends on $\bar{s}^{e q}$ only through a suitable scalar function $\hat{\alpha}$ :

$$
\alpha=\hat{\alpha}\left(\bar{s}^{e q}\right)
$$

\subsection{Complementary law for damage}

The model requires additional equations which govern the evolution of $\alpha$ and these are introduced in the framework of a standard thermodynamic formulation. It is assumed that damage may increase only when $X$ attains a threshold value $X^{0}$ which depends only on the current value of the damage variable $\alpha$, whereby it results:

$$
f=X(\mathbf{s}, \alpha)-X^{0}(\alpha) \leq 0
$$


with the additional classical Kuhn-Tucker loading-unloading conditions:

$$
\dot{\alpha} \geq 0 \quad f \leq 0 \quad \dot{\alpha} f=0
$$

In order to have $\dot{\alpha}>0$, the consistency condition requires not only that $f=0$, but also that $\dot{f}=0$ whereby, with the aid of the chain rule, it results:

$$
\dot{f}=\frac{\partial X}{\partial \alpha} \dot{\alpha}+\frac{\partial X}{\partial \mathbf{s}} \dot{\mathbf{s}}-\frac{d X^{0}}{d \alpha} \dot{\alpha}=0, \quad \text { if } \dot{\alpha}>0
$$

which gives:

$$
\dot{\alpha}=-\left(\frac{\partial X}{\partial \alpha}-\frac{d X^{0}}{d \alpha}\right)^{-1} \frac{\partial X}{\partial \mathbf{s}} \dot{\mathbf{s}}
$$

On the other hand, the evolution of $\alpha$ can be restated in terms of the evolution of the equivalent displacement $\bar{s}^{e q}$ using equation (11), which yields

$$
\dot{\alpha}=\frac{\partial \hat{\alpha}}{\partial \bar{s}^{e q}} \dot{\bar{s}}^{e q}
$$

In order to have $\dot{\alpha}>0$ in equation (16), it must therefore result $\dot{\bar{s}}^{e q} \neq 0$. From the definition of $\bar{s}^{e q}$ in equation (10) this can only happen if $\dot{\bar{s}}^{e q}>0, s^{e q}=\bar{s}^{e q}$ and $\dot{s}^{e q}=\dot{\bar{s}}^{e q}$. Therefore we can write:

$$
\dot{\alpha}=\frac{d \hat{\alpha}}{d s^{e q}} \frac{\partial s^{e q}}{\partial \mathbf{s}} \dot{\mathbf{s}} \quad \text { if } \dot{\alpha}>0
$$

By equating formulas (17) and (15), one obtains:

$$
\frac{d \hat{\alpha}}{d s^{e q}} \frac{\partial s^{e q}}{\partial \mathbf{s}}=-\left(\frac{\partial X}{\partial \alpha}-\frac{d X^{0}}{d \alpha}\right)^{-1} \frac{\partial X}{\partial \mathbf{s}}
$$

which represents a system of two differential equations to be satisfied by the two scalar functions $\hat{\alpha}$ and $X^{0}$.

Remark 2.1. The choice of assuming $X^{0}$ to depend on $\alpha$ only is not compulsory but it is frequent in cohesive interface formulations. It will be seen in the next sections that this choice leads to strong constraints for the input values, and in particular for the fracture energies in modes I and II, to preserve thermodynamic consistency.

Instead, $X^{0}$ could be made to depend on $\mathbf{s}$ too and it is easy to show that this would allow one to introduce the dependence of damage evolution and fracture energy on mode mixity. Another way to introduce the dependence of fracture energy on the mixed-mode ratio can be to formally define two separate damage variables, one for mode I and one for mode II. However, to be physically realistic these damage variables should simultaneously reach the value corresponding to 
full damage. For example Alfano and Crisfield (2001) make the two damage variables evolve in exactly the same way, resulting in the violation of the normality rule for the damage evolution law. In both cases, the procedure is essentially phenomenological and the underlying physics behind the dependence of the measured fracture energy on mode-mixity is hidden within the model equations.

Hence, it is believed that making $X^{0}$ depend on $\alpha$ only is conceptually and practically a valid alternative, because, as it will be shown in the next sections, the dependence on mode mixity can be reintroduced in the model by making use of well justified, simplified micro-mechanical assumptions.

\section{Specialisation of the model to linear elastic constant-damage re- sponse}

In this section, the model formulated in the previous section is specialized to the case in which the following further hypothesis is made:

VI Linear elastic behavior - The interface responds with a linear elastic stress-displacement law when $\alpha$ does not evolve.

Hypothesis IV specializes formulas (5) and (6) as:

$$
\begin{array}{ll}
\mathbf{X}^{(I 0)}=\left\{s_{n}^{0}, 0, \alpha^{(I 0)}\right\}^{t}, & \mathbf{X}^{(I I 0)}=\left\{0, s_{t}^{0}, \alpha^{(I I 0)}\right\}^{t} \\
\mathbf{X}^{(I f)}=\left\{s_{n}^{f}, 0, \alpha^{(I f)}\right\}^{t}, & \mathbf{X}^{(I I f)}=\left\{0, s_{t}^{f}, \alpha^{(I I f)}\right\}^{t}
\end{array}
$$

Because of Hypothesis VI, the Helmholtz free energy function $\Psi$ is required to be a quadratic form in $\mathbf{s}$, admitting the following representation form:

$$
\Psi(\mathbf{s}, \alpha)=\frac{1}{2} K_{n}^{\alpha}(\alpha) s_{n}^{2}+\frac{1}{2} K_{t}^{\alpha}(\alpha) s_{t}^{2}+\frac{1}{2} K_{n t}^{\alpha}(\alpha) s_{n} s_{t}
$$

Hypothesis IV yields that the function $K_{n t}^{\alpha}$ must be equal to zero so that representation (21) simplifies to:

$$
\Psi(\mathbf{s}, \alpha)=\frac{1}{2} K_{n}^{\alpha}(\alpha) s_{n}^{2}+\frac{1}{2} K_{t}^{\alpha}(\alpha) s_{t}^{2}
$$

The values $K_{n}^{\alpha}$ and $K_{t}^{\alpha}$ are the secant stiffness moduli in unloading.

According to equation (22), the stress and the thermodynamic force associated with $\alpha$ are:

$$
\begin{gathered}
\boldsymbol{\sigma}(\mathbf{s}, \alpha)=\frac{\partial \Psi}{\partial \mathbf{s}}=\left\{K_{n}^{\alpha}(\alpha) s_{n}, K_{t}^{\alpha}(\alpha) s_{t}\right\}^{t} \\
X(\mathbf{s}, \alpha)=-\frac{\partial \Psi}{\partial \alpha}=-\frac{1}{2} K_{n}^{\prime}(\alpha) s_{n}^{2}-\frac{1}{2} K_{t}^{\prime}(\alpha) s_{t}^{2}
\end{gathered}
$$


respectively, where

$$
K_{n}^{\prime}=\frac{d K_{n}^{\alpha}}{d \alpha}, \quad K_{t}^{\prime}=\frac{d K_{t}^{\alpha}}{d \alpha}
$$

Equation (18) can be written in a form that explicitly contains the constitutive functions $K_{n}^{\alpha}, K_{t}^{\alpha}$ and $X^{0}$. Actually, upon differentiating (24) one obtains:

$$
\begin{gathered}
\frac{\partial X}{\partial \alpha}=-\frac{1}{2} K_{n}^{\prime \prime}(\alpha) s_{n}^{2}-\frac{1}{2} K_{t}^{\prime \prime}(\alpha) s_{t}^{2} \\
\frac{\partial X}{\partial \mathbf{s}}=-\left\{K_{n}^{\prime} s_{n}, K_{n}^{\prime} s_{n}\right\}^{t}
\end{gathered}
$$

and substitution of (26) and (27) into (18) yields:

$$
\frac{\partial s^{e q}}{\partial \mathbf{s}}=\left\{\begin{array}{l}
\frac{K_{n}^{\prime} s_{n}}{\frac{d \alpha}{d s^{e q}}\left[-\frac{1}{2}\left(K_{n}^{\prime \prime} s_{n}^{2}+K_{t}^{\prime \prime} s_{t}^{2}\right)-\frac{d X^{0}}{d \alpha}\right]} \\
\frac{K_{t}^{\prime} s_{t}}{\frac{d \alpha}{d s^{e q}}\left[-\frac{1}{2}\left(K_{n}^{\prime \prime} s_{n}^{2}+K_{t}^{\prime \prime} s_{t}^{2}\right)-\frac{d X^{0}}{d \alpha}\right]}
\end{array}\right\}
$$

Equation (28) is a partial differential equation that represents a sufficient condition to preserve thermodynamic consistency, to be fulfilled for the existence of $s^{e q}$.

Alongside of (28), boundary conditions for $s^{e q}$ have to be also considered. These are related to the conditions to be satisfied by the interface response at the onset of damage (curve $\boldsymbol{\Gamma}_{0}$ ) and at complete decohesion (curve $\boldsymbol{\Gamma}_{f}$ ).

Specifically, in compliance with (7) and (12), at the onset of damage, in pure mode I and pure mode II, the same damage and same damage-driven force must be attained

$$
\begin{gathered}
\alpha^{(I 0)}=\alpha^{(I I 0)} \\
X^{(I 0)}=X^{(I I 0)}
\end{gathered}
$$

Analogously, at complete decohesion the same damage $\alpha$ and damage-driven force must be attained in pure modes I and II.

$$
\begin{gathered}
\alpha^{(I f)}=\alpha^{(I I f)} \\
X^{(I f)}=X^{(I f f)}
\end{gathered}
$$

Equations (29), (30), (31) and (32) are rewritten below in terms of equivalent displacement by means of (24)

$$
s^{e q}\left(\mathbf{s}^{(I 0)}\right)=s^{e q}\left(\mathbf{s}^{(I I 0)}\right)
$$




$$
\begin{aligned}
\frac{1}{2} K_{n}^{\prime}\left(s^{e q}\left(\mathbf{s}^{(I 0)}\right)\right)\left(s_{n}^{0}\right)^{2} & =\frac{1}{2} K_{t}^{\prime}\left(s^{e q}\left(\mathbf{s}^{(I I 0)}\right)\right)\left(s_{t}^{0}\right)^{2} \\
s^{e q}\left(\mathbf{s}^{(I f)}\right) & =s^{e q}\left(\mathbf{s}^{(I f f)}\right) \\
\frac{1}{2} K_{n}^{\prime}\left(s^{e q}\left(\mathbf{s}^{(I f)}\right)\right)\left(s_{n}^{f}\right)^{2} & =\frac{1}{2} K_{t}^{\prime}\left(s^{e q}\left(\mathbf{s}^{(I I f)}\right)\right)\left(s_{t}^{f}\right)^{2}
\end{aligned}
$$

\subsection{Damage model with linear mode I and mode II softening}

To reproduce an experimentally measured interface response the model requires calibration with an appropriate assignment of the functions $K_{n}^{\alpha}$ and $K_{t}^{\alpha}$.

However, the dependence of the secant moduli as function of $\alpha$ is only provided implicitly by the experimental diagrams. Actually, the secant moduli functions that can be straighforwardly acquired on the basis of the measured, or assumed, stress-displacement mode I (mode II) responses are available in the form of functions whose independent variable is the maximum normal (tangential) displacement.

For monotonically increasing relative displacement components histories, (i.e. along $\boldsymbol{\Gamma}_{I}$ and $\boldsymbol{\Gamma}_{I I}$ under the conditions $s_{n} \geq 0, \dot{s_{n}}>0$ and $s_{t} \geq 0, \dot{s_{t}}>0$ ), these functions, denoted $K_{n}^{\mathbf{S}}, K_{t}^{\mathbf{S}}$, can be represented as follows:

$$
K_{n}^{\mathbf{S}}=K_{n}^{\alpha} \circ \hat{\alpha} \circ s_{I}^{e q}, \quad K_{t}^{\mathbf{S}}=K_{t}^{\alpha} \circ \hat{\alpha} \circ s_{I I}^{e q}
$$

In (37) (o) indicates function composition and function $\hat{\alpha}$ has been introduced by equation (11) while $s_{I}^{e q}$ and $s_{I I}^{e q}$ are functions defined as follows:

$$
s_{I}^{e q}: \quad s_{I}^{e q}\left(s_{n}\right)=s^{e q}\left(s_{n}, 0\right), \quad s_{I I}^{e q}: \quad s_{I I}^{e q}\left(s_{t}\right)=s^{e q}\left(0, s_{t}\right)
$$

For monotonic modes I and II, the functions $s_{I}^{e q}$ and $s_{I I}^{e q}$ are increasing, and hence one-to-one, functions of their arguments. Consequently their inverse functions exist and are denoted as:

$$
s_{n}^{\alpha}=\left(s_{I}^{e q}\right)^{-1} \circ \hat{\alpha}^{-1}, \quad s_{t}^{\alpha}=\left(s_{I I}^{e q}\right)^{-1} \circ \hat{\alpha}^{-1}
$$

According to (39) the function $s_{n}^{\alpha}(\alpha)$ is introduced, such that, for a given $\alpha, s_{n}^{\alpha}(\alpha)$ represents the displacement $s_{n}$ that in a monotonic mode I path corresponds to the first attainment of damage value $\alpha$. Similarly, $s_{t}^{\alpha}(\alpha)$ represents the displacement $s_{t}$ that in a monotonic mode II path corresponds to the first attainment of a level of damage equal to $\alpha$.

Finally, on account of the definition of $s_{n}^{\alpha}$ and $s_{t}^{\alpha}$, it can be inferred from (37):

$$
K_{n}^{\alpha}=K_{n}^{\mathbf{S}} \circ s_{n}^{\alpha}, \quad K_{t}^{\alpha}=K_{t}^{\mathbf{S}} \circ s_{t}^{\alpha}
$$

To consider a specific example, the analytical expressions that yield bilinear stress-strain laws, under monotonically applied increasing displacements in pure 
modes I and II, are now examined. The relation between secant moduli and maximum displacements attained for the softening branches can be obtained by means of simple geometrical considerations. In particular, denoting by $K_{n 0}$ the initial elastic normal stiffness, by $s_{n}^{0}$ the normal displacement at the onset of damage and by $s_{n}^{f}$ the displacement at which zero stiffness is attained, the expression for $K_{n}^{\mathbf{S}}$ is:

$$
K_{n}^{\mathbf{S}}\left(s_{n}\right)=\frac{K_{n 0} s_{n}^{0}}{s_{n}^{f}-s_{n}^{0}}\left(\frac{s_{n}^{f}}{s_{n}}-1\right)
$$

Similarly, in mode II, denoting by $s_{t}^{0}$ the displacement at the onset of damage and by $s_{t}^{f}$ the displacement at which zero stiffness is attained, $K_{t}^{\mathbf{S}}$ turns out to be:

$$
K_{t}^{\mathbf{S}}\left(s_{t}\right)=\frac{K_{t 0} s_{t}^{0}}{s_{t}^{f}-s_{t}^{0}}\left(\frac{s_{t}^{f}}{s_{t}}-1\right)
$$

where $K_{t 0}$ is the initial elastic tangential stiffness. The constitutive functions $K_{n}^{\alpha}$ and $K_{t}^{\alpha}$ are thus

$$
K_{n}^{\alpha}(\alpha)=\frac{K_{n 0} s_{n}^{0}}{s_{n}^{f}-s_{n}^{0}}\left(\frac{s_{n}^{f}}{s_{n}^{\alpha}(\alpha)}-1\right), \quad K_{t}^{\alpha}(\alpha)=\frac{K_{t 0} s_{t}^{0}}{s_{t}^{f}-s_{t}^{0}}\left(\frac{s_{t}^{f}}{s_{t}^{\alpha}(\alpha)}-1\right)
$$

Differentiating (43) with respect to $\alpha$ one obtains

$$
K_{n}^{\prime}(\alpha)=-K_{n 0} \frac{s_{n}^{0} s_{n}^{f}}{s_{n}^{f}-s_{n}^{0}} \frac{1}{s_{n}^{2}} \frac{d s_{n}^{\alpha}}{d \alpha}, \quad K_{t}^{\prime}(\alpha)=-K_{t 0} \frac{s_{t}^{0} s_{t}^{f}}{s_{t}^{f}-s_{t}^{0}} \frac{1}{s_{t}^{2}} \frac{d s_{t}^{\alpha}}{d \alpha}
$$

\subsection{Equivalent displacement function}

The following equivalent displacement function considered by Sacco and Toti (2010) is hereby used:

$$
\begin{gathered}
s^{e q}= \begin{cases}0 & \text { if } \quad \tilde{s}^{e q} \leq 0 \\
\tilde{s}^{e q} & \text { if } \quad 0 \leq \tilde{s}^{e q} \leq 1 \\
1 & \text { if } \quad \tilde{s}^{e q} \geq 1\end{cases} \\
\tilde{s}^{e q}=\frac{\beta-1}{\beta} \frac{1}{1-\eta}
\end{gathered}
$$

where

$$
\beta(\mathbf{s})=\sqrt{\left(\frac{s_{n}}{s_{n}^{0}}\right)^{2}+\left(\frac{s_{t}}{s_{t}^{0}}\right)^{2}}, \quad \eta(\mathbf{s})=\frac{s_{n}^{2}}{\|\mathbf{s}\|^{2}} \frac{s_{n}^{0}}{s_{n}^{f}}+\frac{s_{t}^{2}}{\|\mathbf{s}\|^{2}} \frac{s_{t}^{0}}{s_{t}^{f}}
$$


A computation of $s_{I}^{e q}$ and $s_{I I}^{e q}$ from (46) provides:

$$
s_{I}^{e q}=\frac{s_{n}^{f}}{s_{n}^{f}-s_{n}^{0}}\left(1-\frac{s_{n}^{0}}{s_{n}}\right), \quad s_{I I}^{e q}=\frac{s_{t}^{f}}{s_{t}^{f}-s_{t}^{0}}\left(1-\frac{s_{t}^{0}}{s_{t}}\right)
$$

Taking the inverse of the previous functions, according to (39) one has:

$$
s_{n}^{\alpha}(\alpha)=\frac{s_{n}^{0} s_{n}^{f}}{s_{n}^{f}-\alpha\left(s_{n}^{f}-s_{n}^{0}\right)}, \quad s_{t}^{\alpha}(\alpha)=\frac{s_{t}^{0} s_{t}^{f}}{s_{t}^{f}-\alpha\left(s_{t}^{f}-s_{t}^{0}\right)}
$$

According to (40), combining these last relations with (41) and (42) provides:

$$
K_{n}^{\alpha}=(1-\alpha) K_{n 0}, \quad K_{t}^{\alpha}=(1-\alpha) K_{t 0}
$$

Therefore, the adoption of Sacco and Toti's norm recovers for parameter $\alpha$ the meaning of the ratio of the area $d A_{d}$ of the damaged subset of the interface over the total interface area $d A$, bounded by the limits $0 \leq \alpha \leq 1$. Accordingly, the quantity $(1-\alpha)$ recovers the meaning of the complementary undamaged area fraction.

Differentiation of functions (50) with respect to the dimensionless parameter $\alpha$ finally yields

$$
K_{n}^{\prime}=-K_{n 0}, \quad K_{t}^{\prime}=-K_{t 0}, \quad K_{n}^{\prime \prime}=0, \quad K_{t}^{\prime \prime}=0
$$

Sacco and Toti's model also addresses linear softening branches in pure modes I and II, described by equations (41) and (42). The possibility of retrieving this behavior in fulfillment of thermodynamic consistency is now examined. This requirement specifically amounts, in the light of the previous discussion, to the fulfillment of the domain equation (28) and of boundary conditions (33)-(36).

The fulfillment of boundary conditions by $s^{e q}$ is analyzed first. Function (45) authomatically satisfies (33) and (35) with $\alpha^{0}=0$ and $\alpha^{f}=1$, as it can be easily verified. Hence, it is necessary to satisfy the remaining conditions on the $X$, represented by (34) and (36). In view of (51), these simply specialize to:

$$
\begin{aligned}
\frac{1}{2} K_{n 0}\left(s_{n}^{0}\right)^{2} & =\frac{1}{2} K_{t 0}\left(s_{t}^{0}\right)^{2} \\
\frac{1}{2} K_{n 0}\left(s_{n}^{f}\right)^{2} & =\frac{1}{2} K_{t 0}\left(s_{t}^{f}\right)^{2}
\end{aligned}
$$

Equation (52) states that the same elastic energy must be attained in mode I and mode II at the onset of damage. Moreover, the following constraint for displacements stems from the combination of (52) and (53):

$$
\frac{s_{n}^{f}}{s_{n}^{0}}=\frac{s_{t}^{f}}{s_{t}^{0}}
$$


These constraints are recognized to introduce a quite severe limitation to the possible mechanical behaviors addressed by the model. Actually, a significant restriction imposed by (52) and (54) is recognized by combining these two equations into the following one:

$$
\frac{1}{2} K_{n 0} s_{n}^{0} s_{n}^{f}=\frac{1}{2} K_{t 0} s_{t}^{0} s_{t}^{f}
$$

Equation (55) represents the coincidence of the areas underneath the triangular diagrams of stress-relative displacements laws in modes I and II and, thus, simply equates the fracture energies in mode I, $G_{c}^{n}$, and mode II, $G_{c}^{t}$

$$
G_{c}^{n}=G_{c}^{t}
$$

This requirement is in agreement with the results of Del Piero and Raous (2010). In this reference, it is observed that, as a result of the employment of a single damage variable, one single diagonal experiment is sufficient to determine the constitutive behavior. It is worth recalling that also Alfano and Crisfield (2001) introduce a restriction to specialize the 2 damage parameters model of Crisfield and coworkers Mi et al. (1998) to a 1 damage parameter model so as to achieve simultaneous complete debonding in mode I and II. Similarly, other authors (Allix and Corigliano, 1996; Corigliano, 1993) add this 'simultaneity' condition as a further constraint to the evolution equations.

The second requirement for thermodynamic consistency is represented by the fulfillment of equation (28). According to (51) equations (28) specialize as:

$$
\frac{\partial s^{e q}}{\partial s_{n}}=\frac{K_{n 0} s_{n}}{\frac{d \alpha}{d s^{e q}} \frac{d X^{0}}{d \alpha}}, \quad \frac{\partial s^{e q}}{\partial s_{t}}=\frac{K_{t 0} s_{t}}{\frac{d \alpha}{d s^{e q}} \frac{d X^{0}}{d \alpha}}
$$

Since the denominators of the right hand sides in equations (57) are equal, the following equation is inferred from (57) as a necessary condition for the existence of a function $X^{0}(\alpha)$ capable of fulfilling (28):

$$
\frac{K_{n 0} s_{n}}{\frac{\partial s^{e q}}{\partial s_{n}}}=\frac{K_{t 0} s_{t}}{\frac{\partial s^{e q}}{\partial s_{t}}}
$$

The components of $\frac{\partial s^{e q}}{\partial \mathbf{s}}$ appear at the denominator of the previous equation. The explicit expressions for these components are written below with the usual aid of the chain rule:

$$
\frac{\partial s^{e q}}{\partial \mathbf{s}}=\frac{\partial s^{e q}}{\partial \beta} \frac{\partial \beta}{\partial \mathbf{s}}+\frac{\partial s^{e q}}{\partial \eta} \frac{\partial \eta}{\partial \mathbf{s}}
$$

The terms in the previous equations are

$$
\frac{\partial s^{e q}}{\partial \beta}=\frac{1}{\beta^{2}(1-\eta)}, \quad{ }_{13} \quad \frac{\partial s^{e q}}{\partial \eta}=\frac{\beta-1}{\beta(1-\eta)^{2}}
$$


and

$$
\begin{gathered}
\frac{\partial \beta}{\partial \mathbf{s}}=\frac{1}{\beta}\left\{\begin{array}{c}
\frac{s_{n}}{\left(s_{n}^{0}\right)^{2}} \\
\frac{s_{t}}{\left(s_{t}^{0}\right)^{2}}
\end{array}\right\} \\
\frac{\partial \eta}{\partial \mathbf{s}}=\frac{2}{\|\mathbf{s}\|^{2}}\left(\left\{\begin{array}{c}
\frac{s_{n}^{0} s_{n}}{s_{n}^{f}} \\
\frac{s_{t}^{0} s_{t}}{s_{t}^{f}}
\end{array}\right\}-\eta \mathbf{s}\right)
\end{gathered}
$$

The overall gradient thus turns out to be

$$
\frac{\partial s^{e q}}{\partial \mathbf{s}}=\frac{1}{\beta^{3}(1-\eta)}\left\{\begin{array}{c}
\frac{s_{n}}{\left(s_{n}^{0}\right)^{2}} \\
\frac{s_{t}}{\left(s_{t}^{0}\right)^{2}}
\end{array}\right\}+\frac{\beta-1}{\beta(1-\eta)^{2}}\left(\frac{2}{\|\mathbf{s}\|^{2}}\left(\left\{\begin{array}{c}
\frac{s_{n}^{0} s_{n}}{s_{n}^{f}} \\
\frac{s_{t}^{0} s_{t}}{s_{t}^{f}}
\end{array}\right\}-\eta \mathbf{s}\right)\right)
$$

Substituting (51) and (63) in the denominators of (58) one arrives to the conclusion that the displacement defined by expressions (46) and (47) does not generally admit a thermodynamic consistent reformulation, except for special cases. Actually, satisfaction of (58) is only guaranteed if the following conditions are fulfilled

$$
K_{n 0}=K_{t 0}, \quad s_{n}^{0}=s_{t}^{0}, \quad s_{n}^{f}=s_{t}^{f}
$$

Since the fulfillment of (64) implies the fulfillment of (52) and (53), conditions (64) represent a stronger limitation to the interface model.

On the other hand, in the next two sections it will be shown that these limitations can be overcome by enhancing the cohesive-zone model to account for two important aspects of the physics of the problem, namely friction and interlocking, the latter being related to the roughness of the fracture interface. Rather than doing so using a pure phenomenological approach, friction and interlocking are introduced in the model based on simplified, yet effective, micro-mechanical arguments.

\section{Frictional damage model}

In this section the opening-sliding damage model of the previous section, endowed with the equivalent displacement norm of subsection 3.2, is combined with unilateral contact and friction exploiting the strategy employed, among others, by Alfano and Sacco (2006) and Spada et al. (2009). The state of the surface, 
apart from the displacement $\mathbf{s}$, is completely defined by the history variables $\alpha$ and a new internal variable $s_{p t}$ representing friction sliding in tangential direction. This last parameter accounts for inelastic sliding which acts only in tangential direction and enters the model in the form of nonassociative plasticity.

Accordingly, the free energy $\Psi$ is decomposed as the sum of the elastic energy on the undamaged and damaged parts of such plane, denoted by $\Psi_{u}$ and $\Psi_{d}$, respectively:

$$
\Psi\left(\mathbf{s}, \alpha, s_{p t}\right)=(1-\alpha) \Psi_{u}(\mathbf{s})+\alpha \Psi_{d}\left(\mathbf{s}, s_{p t}\right)
$$

For the specific free energy of the undamaged part, the following quadratic expression, associated with a linear elastic stress-relative displacement relation, is considered:

$$
\Psi_{u}=\frac{1}{2}\left(K_{n 0} s_{n}^{2}+K_{t 0} s_{t}^{2}\right)
$$

The expression of $\Psi_{d}$ is devised so as to account for unilateral contact in the normal direction and friction, in the form of a tangential frictional slip $s_{p t}$ :

$$
\Psi_{d}=\frac{1}{2}\left[K_{n 0}<s_{n}>_{-}^{2}+K_{t 0}\left(s_{t}-s_{p t}\right)^{2}\right]
$$

where the brackets $\langle\bullet\rangle_{-}$denote the function that extracts the negative part of the argument $\langle x\rangle_{-}=(x-|x|) / 2$.

The governing set of equations is completed by the equations that describe the evolution of the history parameters $\alpha$ and $s_{p t}$.

The evolution law for $\alpha$ is the same as the one that has been discussed in the previous section.

As for frictional sliding, the following threshold function of the Mohr Coulomb type, with no cohesion, is considered :

$$
\phi\left(\boldsymbol{\sigma}_{d}\right)=\mu<\sigma_{d n}>_{-}+\left|\sigma_{d t}\right|
$$

where

$$
\boldsymbol{\sigma}_{d}=\frac{\partial \Psi_{d}}{\partial \mathbf{s}}, \quad \sigma_{d n}=\frac{\partial \Psi_{d}}{\partial s_{n}}, \quad \sigma_{d t}=\frac{\partial \Psi_{d}}{\partial s_{t}}
$$

Frictional slip is obtained by means of a slip function $g\left(\boldsymbol{\sigma}_{d}\right)$ and postulating that it results

$$
\dot{\mathbf{s}}_{p}=\dot{\lambda} \frac{\partial g}{\partial \boldsymbol{\sigma}_{d}}
$$

where $\lambda$, similar to the plastic multiplier introduced in elastoplasticity by Simo and Hughes (1998), is the slip multiplier. To address a pure frictional effect with no dilatancy, the following slip potential is considered:

$$
g\left(\boldsymbol{\sigma}_{d}\right)=\left|\sigma_{d n}\right|
$$


The slip potential (71) provides an entirely tangential flow rule:

$$
\dot{s}_{p t}=\dot{\lambda} \frac{\partial \hat{g}}{\partial \sigma_{d t}}=\dot{\lambda} \operatorname{sign}\left(\sigma_{d t}\right), \quad \dot{s}_{p n}=0
$$

which is completed by the additional Khun-Tucker conditions:

$$
\dot{\lambda} \geq 0, \quad \phi \leq 0 \quad \dot{\lambda} \phi\left(\sigma_{d}\right)=0
$$

Summarizing, the evolution of the tangential frictional slip $s_{p t}$ is governed by the system of nonlinear coupled differential equations, (68), (72) and (73).

The frictional damage evolution law by Alfano and Sacco (2006) summarised in this section correctly leads to an increase in total energy dissipated, i.e. macroscopically measured fracture energy, due to the frictional dissipation when mode II is accompanied by compressive stress in mode I. However, for pure mode II without compression in mode I the model would predict no frictional dissipation and therefore no increase in fracture energy. This is in contrast with the experimental finding that, for most material interfaces, a significant increase in fracture energy is measured for increasing mode II/mode I ratios even for positive relative displacements in mode I. In the next section it is shown how this shortcoming can be overcome by further enhancing the model to account for interface roughness and associated interlocking mechanism.

\section{Enhanced micromechanics of rough interface with interlocking}

As shown in the previous sections, use of a single damage variable in a cohesive zone model for quasi brittle materials, when combined with the choice of employing an equivalent relative displacement, introduces several limitations for the damage model, and specifically requires coincidence of fracture energies in modes I and II to comply with thermodynamic consistency. This result is in agreement with past theoretical (Del Piero and Raous, 2010) and experimental (Carpinteri et al., 1993) researches on cohesive zone interface models, that have raised questions on the viability of treating mode II fracture energy as a real material parameter independent from mode I fracture energy.

Focusing in particular on concrete, experiments in mixed mode crack propagation typically show an apparent mode II fracture energy even up to $30 \%$ higher than its mode I counterpart (Carpinteri et al., 1993). Therefore, it is desirable to have interface models capable of accounting for this experimental evidence.

To bridge the theoretical and engineering issues posed by these two seemingly opposite evidences, a meso-scale cohesive zone model is considered. In particular, the solution exploited for retrieving a greater fracture energy in mode-II consists of a simplified meso-scale approach based on the enhancement proposed by Ser-

pieri and Alfano (2011) whereby the geometry of the interface is represented in the 
form of a periodic arrangement of distinct inclined planes, denominated Representative Interface Element (RIE). The interaction within each of these surfaces is governed by the combined damage-friction interface formulation above discussed.

In the present section the original idea proposed by Serpieri and Alfano (2011) is briefly recalled. The geometry of the interface is represented in the form of a periodic arrangement of distinct inclined planes, denominated Representative Interface Element (RIE).

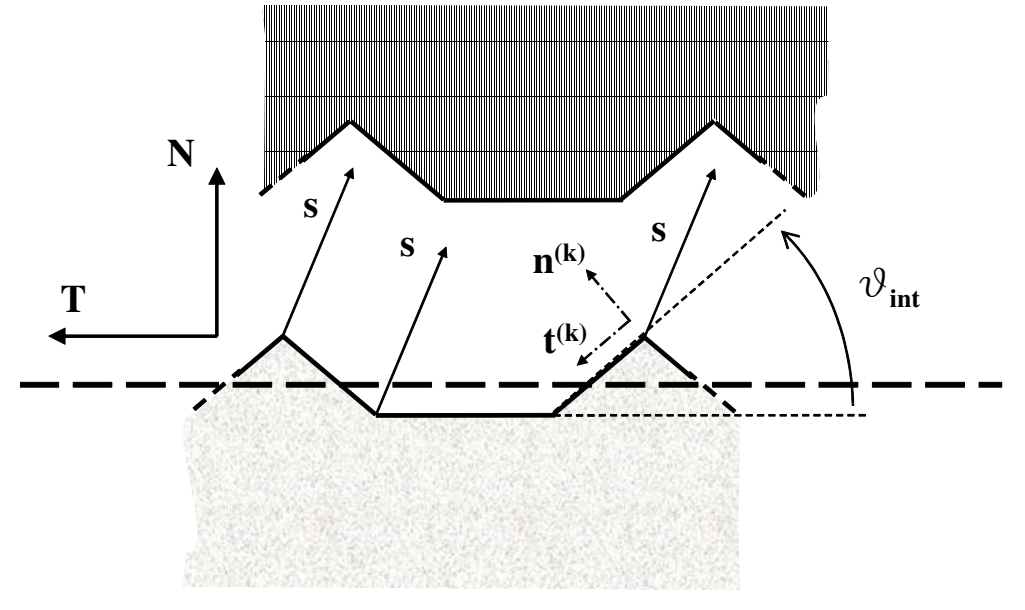

Figure 2: Trapezoidal Representative Interface Element

Figure 2 shows a trapezoidal RIE. The global reference frame $\{N, T\}$ is now introduced on the meso-scale average surface, $N$ and $T$ being the normal and tangential directions of the macroscopic average interface $\mathcal{S}$, shown in Figure 2 by a dotted line. On each $k$-th inclined plane a right handed local coordinate system $n^{(k)}-t^{(k)}$ is also introduced. The unit vectors associated with the axes of the global frame are $\mathbf{N}$ and $\mathbf{T}$, while their counterparts for the $k$-th inclined plane are $\mathbf{n}^{(k)}$ and $\mathbf{t}^{(k)}$.

At any point of the interface, the macroscopic displacement vector $\mathbf{s}$ is considered to be coincident with the displacement vector on each inclined plane $\mathbf{s}^{(k)}$, i.e. $\mathbf{s}^{(k)}=\mathbf{s}$. In the global and local reference frames the overall relative displacement vector $\mathbf{s}$ is represented by the arrays $\{\mathbf{s}\}=\left\{s_{N}, s_{T}\right\}^{t}$ and $\{\mathbf{s}\}^{(k)}=\left\{s_{n}^{(k)}, s_{t}^{(k)}\right\}^{t}$, respectively, with $s_{N}=\mathbf{s} \cdot \mathbf{N}, s_{T}=\mathbf{s} \cdot \mathbf{T}, s_{n}^{(k)}=\mathbf{s} \cdot \mathbf{n}^{(k)}$ and $s_{t}^{(k)}=\mathbf{s} \cdot \mathbf{t}^{(k)}$.

On each $k$-th plane, the internal variables are the damage variable $\alpha^{(k)}$ and the tangential frictional slip $s_{p t}^{(k)}$ which both evolve independently from the inelastic parameters on the other inclined planes. The interaction within each of these sub-surfaces is governed by the combined damage-friction interface formulation presented in the two previous sections. Evolution of $\alpha^{(k)}$ is defined by the equivalent displacement function $s^{e q}$ whose argument on each plane is $\{\mathbf{s}\}^{(k)}$, i.e. 
$\alpha^{(k)}=s^{e q}\left(s_{n}^{(k)}, s_{t}^{(k)}\right)$. Moreover, for each plane, constraints (64), that stem from the requirement of thermodynamic consistency and result in coincidence of mode I and mode II fracture energies as pointed out in section 2 , are fully taken into account.

The free energy per unit area $\Psi$ is defined as the weighted sum of the free energies associated with each contact plane (Serpieri and Alfano, 2011) the weight being the area fraction $\gamma^{(k)}$ i.e. the ratio between the length of the $k$-th inclined plane and the total length of the RIE:

$$
\Psi=\sum_{k=1}^{N^{m}} \gamma^{(k)} \Psi^{(k)}
$$

where $\Psi^{(k)}$ is the free energy per unit area of the $k$-th contact plane.

The macroscopic stress turns out to be expressed as the weighted sum of the contribution of each inclined plane

$$
\boldsymbol{\sigma}=\frac{\partial \Psi}{\partial \mathbf{s}}=\sum_{(k)} \gamma^{(k)} \boldsymbol{\sigma}^{(k)} \quad \text { where } \boldsymbol{\sigma}^{(k)}=\frac{\partial \Psi^{(k)}}{\partial \mathbf{s}}
$$

Stress is in turn divided into two parts associated with the damaged and undamaged parts of the specific free energy:

$$
\boldsymbol{\sigma}^{(k)}=\frac{\partial \Psi^{(k)}}{\partial \mathbf{s}}=\left(1-\alpha^{(k)}\right) \boldsymbol{\sigma}_{u}^{(k)}+\alpha^{(k)} \boldsymbol{\sigma}_{d}^{(k)}, \quad \boldsymbol{\sigma}_{u}^{(k)}=\frac{\partial \Psi_{u}^{(k)}}{\partial \mathbf{s}}, \quad \boldsymbol{\sigma}_{d}^{(k)}=\frac{\partial \Psi_{d}^{(k)}}{\partial \mathbf{s}}
$$

Symbols $\sigma_{N}$ and $\sigma_{T}$ denote the normal and tangential components of the macroscopic stress in the global frame. Conversely, symbols $\sigma_{N}^{(k)}$ and $\sigma_{T}^{(k)}$ are the stresses components on the $k$-th inclined plane, again in global coordinates:

$$
\{\boldsymbol{\sigma}\}=\frac{\partial \Psi}{\partial\left(s_{N}, s_{T}\right)}=\left\{\begin{array}{c}
\sigma_{N} \\
\sigma_{T}
\end{array}\right\}, \quad\left\{\boldsymbol{\sigma}^{(k)}\right\}=\frac{\partial \Psi^{(k)}}{\partial\left(s_{N}, s_{T}\right)}=\left\{\begin{array}{c}
\sigma_{N}^{(k)} \\
\sigma_{T}^{(k)}
\end{array}\right\}
$$

Furthermore, $\sigma_{n}^{(k)}$ and $\sigma_{t}^{(k)}$ denote the normal and tangential stress components contributed on the $k$-th inclined plane with respect to the local system, defined as

$$
\sigma_{n}^{(k)}=\frac{\partial \Psi^{(k)}}{\partial s_{n}^{(k)}}, \quad \sigma_{t}^{(k)}=\frac{\partial \Psi^{(k)}}{\partial s_{t}^{(k)}}
$$

and collected in the vector

$$
\left\{\boldsymbol{\sigma}^{(k)}\right\}^{(k)}=\frac{\partial \Psi^{(k)}}{\partial\left(s_{n}^{(k)}, s_{t}^{(k)}\right)}=\left\{\begin{array}{c}
\sigma_{n}^{(k)} \\
\sigma_{t}^{(k)}
\end{array}\right\}=\left(1-\alpha^{(k)}\right)\left\{\boldsymbol{\sigma}_{u}^{(k)}\right\}^{(k)}+\alpha^{(k)}\left\{\boldsymbol{\sigma}_{d}^{(k)}\right\}^{(k)}
$$


where:

$$
\left\{\boldsymbol{\sigma}_{u}^{(k)}\right\}^{(k)}=\frac{\partial \Psi_{u}^{(k)}}{\partial\left(s_{n}^{(k)}, s_{t}^{(k)}\right)}=\left[\begin{array}{c}
\sigma_{u n}^{(k)} \\
\sigma_{u t}^{(k)}
\end{array}\right], \quad\left\{\boldsymbol{\sigma}_{d}^{(k)}\right\}^{(k)}=\frac{\partial \Psi_{d}^{(k)}}{\partial\left(s_{n}^{(k)}, s_{t}^{(k)}\right)}=\left[\begin{array}{c}
\sigma_{d n}^{(k)} \\
\sigma_{d t}^{(k)}
\end{array}\right]
$$

The stress components on the inclined planes are related to the global stress components by the coordinate change stemming from the chain rule

$$
\left\{\boldsymbol{\sigma}^{(k)}\right\}=\frac{\partial \Psi^{(k)}}{\partial\left(s_{N}, s_{T}\right)}=\frac{\partial \Psi^{(k)}}{\partial\left(s_{n}^{(k)}, s_{t}^{(k)}\right)} \frac{\partial\left(s_{n}^{(k)}, s_{t}^{(k)}\right)}{\partial\left(s_{N}, s_{T}\right)}=[\mathbf{Q}]\left\{\boldsymbol{\sigma}^{(k)}\right\}^{(k)}
$$

where $[\mathbf{Q}]$ is the orthogonal matrix associated with the intrinsic coordinate change tensor $\mathbf{Q}$, such that $[\mathbf{Q}]=[\mathbf{Q}]^{-t}$ and defined as:

$$
[\mathbf{Q}]=\left[\begin{array}{ll}
\frac{\partial s_{N}}{\partial s_{n}^{(k)}} & \frac{\partial s_{N}}{\partial s_{t}^{(k)}} \\
\frac{\partial s_{T}}{\partial s_{n}^{(k)}} & \frac{\partial s_{T}}{\partial s_{t}^{(k)}}
\end{array}\right]=\left[\begin{array}{cc}
\frac{\partial s_{n}^{(k)}}{\partial s_{N}} & \frac{\partial s_{t}^{(k)}}{\partial s_{N}} \\
\frac{\partial s_{n}^{(k)}}{\partial s_{T}} & \frac{\partial s_{t}^{(k)}}{\partial s_{T}}
\end{array}\right]
$$

The coordinate change (81) may be synthetically represented as:

$$
\boldsymbol{\sigma}^{(k)}=\sigma_{n}^{(k)} \mathbf{n}^{(k)}+\sigma_{t}^{(k)} \mathbf{t}^{(k)}
$$

and, obviously, analogous transformations hold for the remaining stress vectors introduced so far:

$$
\boldsymbol{\sigma}_{u}^{(k)}=\sigma_{u n}^{(k)} \mathbf{n}^{(k)}+\sigma_{u t}^{(k)} \mathbf{t}^{(k)}, \quad \boldsymbol{\sigma}_{d}^{(k)}=\sigma_{d n}^{(k)} \mathbf{n}^{(k)}+\sigma_{d t}^{(k)} \mathbf{t}^{(k)},
$$

On each plane the constitutive problem can be thus integrated in the local frame, to provide $\left\{\boldsymbol{\sigma}^{(k)}\right\}^{(k)}$, and the macroscopic stress array $\{\boldsymbol{\sigma}\}$ can be reconstructed in global coordinates according to the following formula

$$
\{\boldsymbol{\sigma}\}=\sum_{k=1}^{N^{m}}[\mathbf{Q}]\left\{\boldsymbol{\sigma}^{(k)}\right\}^{(k)}
$$

\section{Numerical applications}

This section deals with the development of numerical applications. Subsection 6.1 provides some details concerning the developed numerical algorithm for time integration of the interface evolutive equations. Next, Subsection 6.2 illustrates a sensitive analysis of the response of the single interface point. Finally, the last subsection, 6.3, reports the results of a structural simulation with a numericalexperimental comparison. 


\subsection{Numerical procedure}

The overall set of evolutive equations is summarized to derive the finite step relationships. This set is composed of relations (79) and (85) supplemented by the equations describing the evolution of the state parameters associated with friction and damage on each $k$-th plane. In particular, each $k$-th plane contributes two systems of evolutive equations $\mathcal{S}_{F}^{(k)}, \mathcal{S}_{D}^{(k)}$, respectively describing the evolution of the state parameters associated with friction and damage on such plane. System $\mathcal{S}_{F}^{(k)}$, in the unknowns $\sigma_{d t}^{(k)}, \sigma_{d n}^{(k)}, \lambda^{(k)}$, corresponds to equations (68)-(73), relevant to the $k$-th plane, and system $\mathcal{S}_{D}^{(k)}$, in the unknown $\alpha^{(k)}$, corresponds to equations (10), (11), (45)-(47), again for the $k$-th plane.

The path-dependent rate equations of the interface model are converted into step-wise path-independent laws driven by relative displacements $\mathbf{s}$ by selecting a suitable time interpolation for the state variables plus a discretised form of the evolutive equations governing the local response. Specifically, the plastic variables are updated assuming a linear interpolation of $\mathbf{s}$ and $\lambda^{(k)}$, so that $\Delta \mathbf{s}=\Delta t \dot{\mathbf{s}}$ and $\Delta \lambda^{(k)}=\Delta t \dot{\lambda}^{(k)}$, $\dot{\mathbf{s}}$ and $\dot{\lambda}^{(k)}$ entering the finite step problem as constants. Concerning the time advancing scheme, an implicit backward Euler scheme is exploited.

The resulting finite step local problem for a given time interval between the instants $t$ and $t+\Delta t$ consists in determining the updated value of the history variables at time $t+\Delta t$ as function of the local state at time $t$ and of the associated deformation.

An advantage of the presented interface formulation is that the response of each micro-plane is uncoupled from the response on the remaining planes so that on each plane the updating of the state can be performed independently from the variables associated with the other planes. Moreover, on each plane, uncoupling between the evolution equations of damage and inelastic slip allows independent updating of the relevant damage and friction variables.

Therefore, in a typical iteration, an updated value of $\alpha^{(k)}$ at the end of the step $\alpha_{t+\Delta t}^{(k)}$ can be directly computed in explicit form from the discrete counterparts of equations (10), (11), (45)-(47). Next, using Equation (79) the total stress on microplane $k$ is given by:

$$
\left\{\boldsymbol{\sigma}^{(k)}\right\}_{t+\Delta t}^{(k)}=\left(1-\alpha^{(k)}\right)\left\{\boldsymbol{\sigma}_{u}^{(k)}\right\}_{t+\Delta t}^{(k)}+\alpha^{(k)}\left\{\boldsymbol{\sigma}_{d}^{(k)}\right\}_{t+\Delta t}^{(k)}
$$

To compute $\left\{\boldsymbol{\sigma}_{d}^{(k)}\right\}_{t+\Delta t}^{(k)}$, the solution of the discretized finite step counterpart of equations $\mathcal{S}_{F}^{(k)}$ is required. Such solution is obtained by computing first the variation of the plastic multiplier $\Delta \lambda^{(k)}$. It is worth recalling that if $s_{n}^{(k)}<0$, which amounts to the surfaces of microplane $(k)$ persisting in a contact state, 
$\Delta \lambda^{(k)}$ is given by

$$
\Delta \lambda^{(k)}=\frac{1}{K_{t}}\left[\mu K_{n} \Delta s_{n}^{(k)}+\operatorname{sgn} \sigma_{t d, t+\Delta t}^{(k)} K_{t} \Delta s_{t}^{(k)}\right]
$$

whereby the frictional slip is updated as

$$
s_{p t, t+\Delta t}^{(k)}=s_{p t, t}^{(k)}+\Delta \lambda^{(k)} \operatorname{sign}\left(\sigma_{t d, t+\Delta t}^{(k)}\right)
$$

Further details on the integration procedure and the formulae of the tangent operator can be found in Serpieri and Alfano (2011).

\subsection{Constitutive response at the single interface point}

This subsection reports the results of a numerical analysis to assess the capability of the model described in the previous sections to capture the variation of apparent fracture energy in mixed mode loading at the constitutive level for the single interface point. Specifically, it is shown that endowing the cohesive model with the friction effect and considering the presence of inclined planes at a micro-mechanical scale, a greater apparent fracture energy is recovered in mode II, as an effect of friction and interlocking, even when mode I and II fracture energies, stiffness moduli and stresses at damage onset are set equal.

The experimental data reported by Carpinteri et al. (1993) are employed as a term for analysis and assessment of the approach with inclined planes, to validate its viability in providing reasonable estimates of variations of apparent fracture energy in mixed mode loading for concrete (i.e. concrete-to-concrete) interfaces. In this reference mixed mode crack propagation and fracture energy variations are investigated experimentally in four point shear tests for two different concretes made with maximum aggregate sizes of $10 \mathrm{~mm}$ (concrete 1) and $20 \mathrm{~mm}$ (concrete 2 ). As reported, for concrete 1 the (mode I) fracture energy is equal to 0.122 $K J / m^{2}$ and, for concrete 2 , it is equal to $0.145 \mathrm{KJ} / \mathrm{m}^{2}$. These experimental values, (used as input data for the $\mathrm{FE}$ analyses carried out by Carpinteri et al. (1993)) are employed in the present subsection to calibrate the constitutive parameters in the analyses of the local response at the single typical interface point. Crack propagation is not investigated in this subsection. Accordingly, we set $G_{c n}=G_{c t}=0.122 \mathrm{KJ} / \mathrm{m}^{2}$ for concrete interface 1 and $G_{c n}=G_{c t}=$ $0.145 \mathrm{KJ} / \mathrm{m}^{2}$ for concrete interface 2 . In compliance with constraints (64), equal values for $\sigma_{o n}$ and $\sigma_{o t}$ are taken, as reported in Table 1 , as well as equal values of the ratios in equation (54). The common value adopted for the reciprocal of these ratios, $R_{\eta}=\frac{s_{n}^{0}}{s_{n}^{f}}=\frac{s_{t}^{0}}{s_{t}^{f}}$, is also reported in Table 1 .

In all numerical examples the trapezoidal shaped RIE of Figure 2 is employed. The micro-scale pattern is composed of three planes, marked $0, A$ and $B$, with 


\begin{tabular}{|l|l|l|}
\hline \hline$\sigma_{\text {on }}$ & $\sigma_{o t}$ & $R_{\eta}$ \\
\hline $2.4 \mathrm{MPa}$ & $2.4 \mathrm{MPa}$ & 0.95 \\
\hline \hline
\end{tabular}

Table 1: Material parameters common to concrete interfaces 1 and 2 employed in the sensitivity analyses of mixed mode fracture energy.

equal area fractions $\gamma^{(0)}=\gamma^{(A)}=\gamma^{(B)}=0.333$ whose outward normal vectors have respectively inclination angles equal to $0^{\circ},-\theta_{\text {int }}$ and $\theta_{\text {int }}$, where $\theta_{\text {int }}$ is an angle that accounts for interlocking and is measured counterclockwise from the average interface surface.

Mixed mode response is tested by applying a displacement controlled opening separation law in a direction $\mathbf{e}_{l}$, inclined by an angle $\theta_{l}$ from the outward normal direction so that when $\theta_{l}=0^{\circ}, \theta_{l}=90^{\circ}$ pure mode I loading and pure mode II loading are recovered, respectively. The relative displacement orthogonal to $\mathbf{e}_{l}$ is left as a free unloaded degree of freedom.

The response is first investigated for concrete 1 in terms of $s_{l}-\tau_{l}$ diagrams, where $s_{l}=\mathbf{s} \cdot \mathbf{e}_{l}$ and $\tau_{l}=\mathbf{s} \cdot \mathbf{e}_{l}$ are, respectively, the components of relative displacement and stress along the $\mathbf{e}_{l}$ direction.

Figure 3 shows families of $s_{l}-\tau_{l}$ plots generated by varying $\theta_{l}$ in the range $\left[0^{\circ}, 90^{\circ}\right]$ associated with several friction and interlocking angles. In the legends the fracture energies $G_{c l}$ spent in bringing interfaces to complete decohesion is also reported. It is worth observing, in particular from the upper left figure, that when $\mu=0$ the diagrams experiment a moderate variation from mode I bilinear shape and, notably, no variation emerges in fracture energy which is constantly equal to $G_{c l}=0.122 \mathrm{KJ} / \mathrm{m}$. Notice that this mode insensitivity of fracture energy is detected, irrespective of $\theta_{i n t}$, as a peculiar effect of absence of friction.

Figure 4 shows the polar diagrams of fracture energy as function of $\theta_{\text {int }}$ and $\mu$ for concrete 1 . From this figure it can be observed that when $\mu=0$ the polar plot recovers a circumference while fracture energy ratio $R_{G}=\frac{G_{c t}}{G_{c n}}$ increases with both $\theta_{\text {int }}$ and $\mu$.

The trends shown by the analytical sensitivity analyses are in agreement with the experimentally evaluated variations of $R_{G}$ obtained by Carpinteri et al. (1993) where it is found mixed mode fracture energy higher than mode I fracture energy and, specifically, a relative increase of, approximately, 16\% for concrete 1 and $33 \%$ for concrete 2 with larger aggregates. These experimental results were also used to determine quantitatively the interlocking angle, which is, with friction, the remaining unset parameter, by selecting it so as to fit the reported values for $R_{G}$. In particular, the coefficient of sliding friction is taken equal to 1.4 which is the values suggested in ACI code (2005) for concrete placed monolithically. Following a rationale similar to Serpieri and Alfano (2011), the interlocking angle is treated as a mean macroscopic measure of the protruding patterns due to aggregates, 

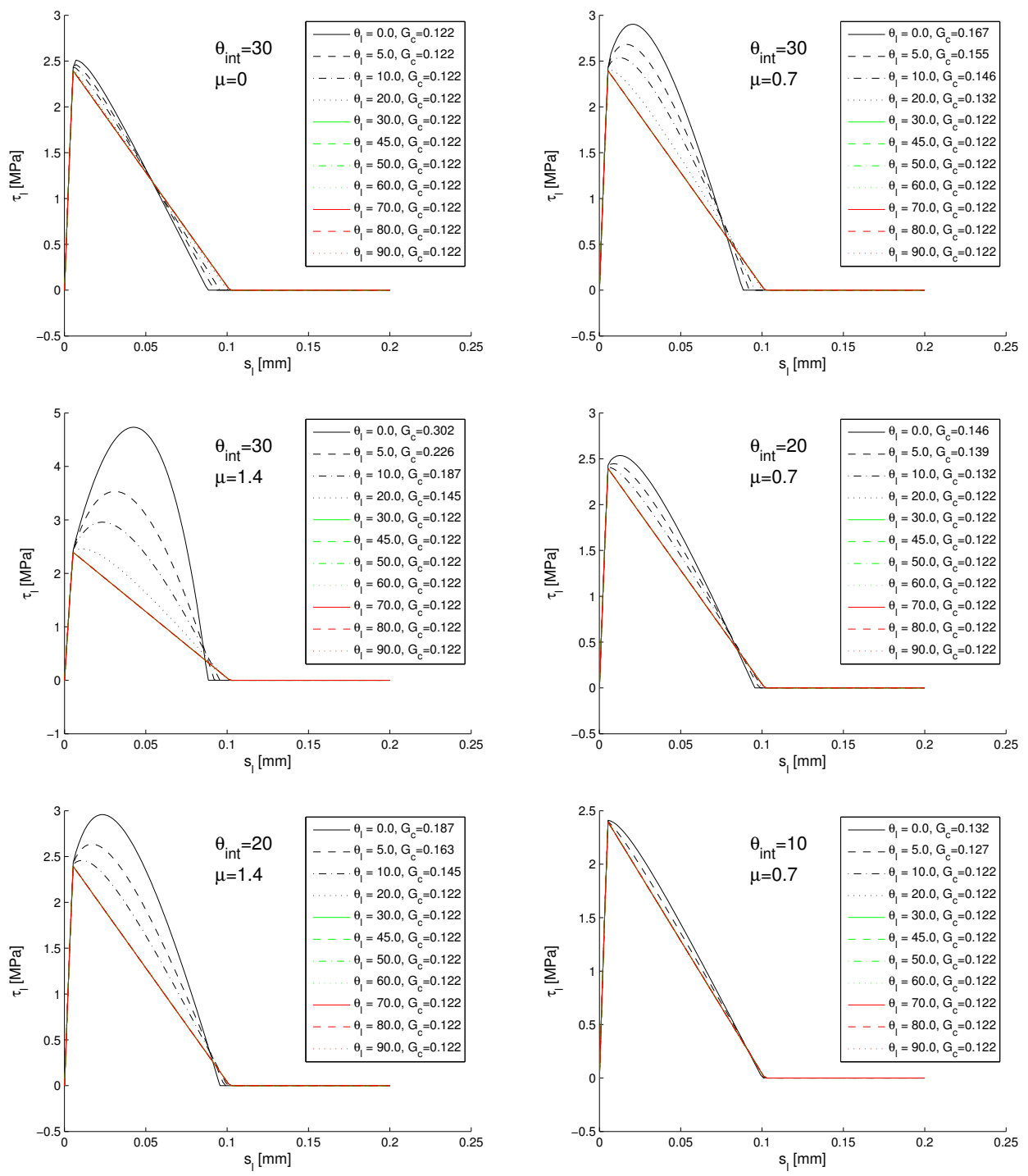

Figure 3: Mixed mode $s_{l}-\tau_{l}$ diagrams for varying $\theta_{\text {int }}$ and $\mu$.

analogously to friction and dilation angles. This match provides $\theta_{\text {int }}=8.5^{\circ}$ for smaller aggregates and $\theta_{\text {int }}=15.0^{\circ}$ for concrete 2 with larger aggregates. Figure 5 shows the contour plot of the numerically computed relation between $R_{G}, \mu$ and $\theta_{\text {int }}$. The two dashed lines are the contour levels corresponding to the values of $R_{G}$ reported by Carpinteri et al. (1993) for concrete 1 (smaller aggregates) and concrete 2 (larger aggregates). The dots denote the points of the $\mu-\theta_{\text {int }}$ plane fitting the experimental values, inferred assuming $\mu=1.4$. 


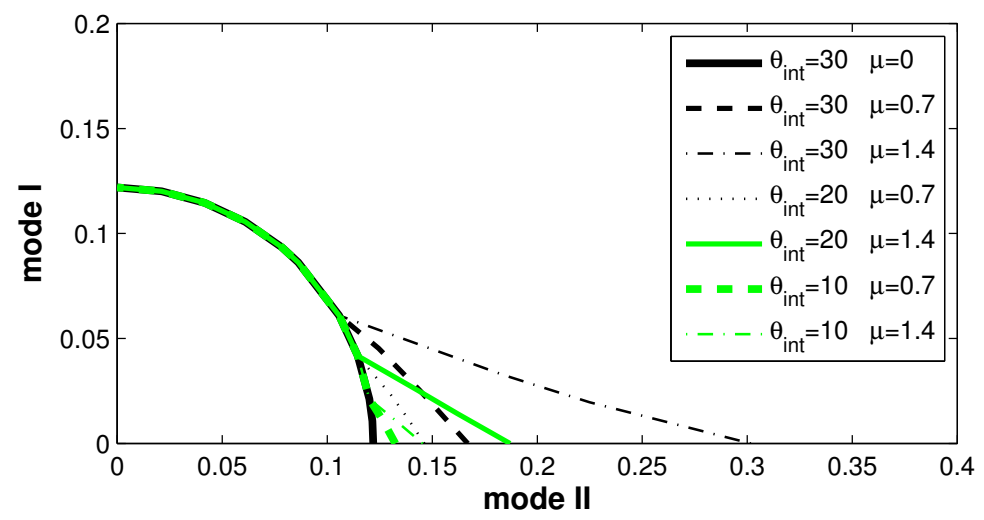

Figure 4: Polar diagrams of fracture energy as function of $\theta_{\text {int }}$ and $\mu$.

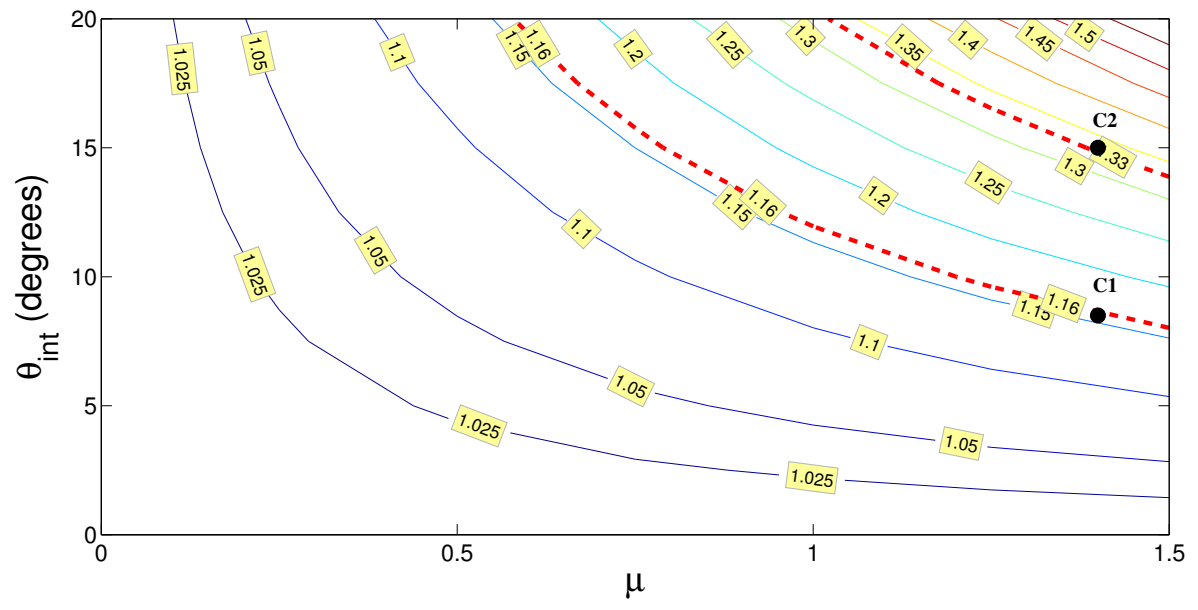

Figure 5: Contour levels of the numerically computed relation between fracture energy ratio $R_{G}$, the tangent of the friction angle, $\mu$, and the interlocking angle $\theta_{\text {int }}$. The dashed lines are the contour levels corresponding to the values of $R_{G}$ reported by Carpinteri et al. (1993) for concrete 1 (smaller aggregates) and concrete 2 (larger aggregates). Dots denote the points of the $\mu-\theta_{\text {int }}$ plane fitting the experimental values, inferred assuming $\mu=1.4$.

\section{3. $D C B-U B M$ Structural simulations}

Nonlinear Finite-Element (FE) simulations of quasi-static structural response were carried out using the commercial2任 $\mathrm{E}$ code Abaqus (2012) (version 6.12-2) 
upon implementing the present interface formulation as a user-defined routine (UMAT) defining the constitutive law for interface elements. In particular, computations concerned the study of the Double Cantilever Beam loaded with Uneven Bending Moments (DCB-UBM), experimentally investigated by Sørensen et al. (2006). The DCB-UBM test has been employed by Sørensen and Jacobsen (2009) to obtain mode mixity-dependent experimental measurements of the crack tip fracture energy. These experimental data provide a suitable term of comparison to assess the capability of the present interface model in predicting, also at the structural level, the increase in fracture energy under increasing mode II loading.

The specimens tested by Sørensen and Jacobsen considered in the simulations are composite beams made of E-glass laminates in polyester matrix, whose geometry, constraints and loading are schematically reported in Figure 6 . The out-of-plane width is $B=30 \mathrm{~mm}$.

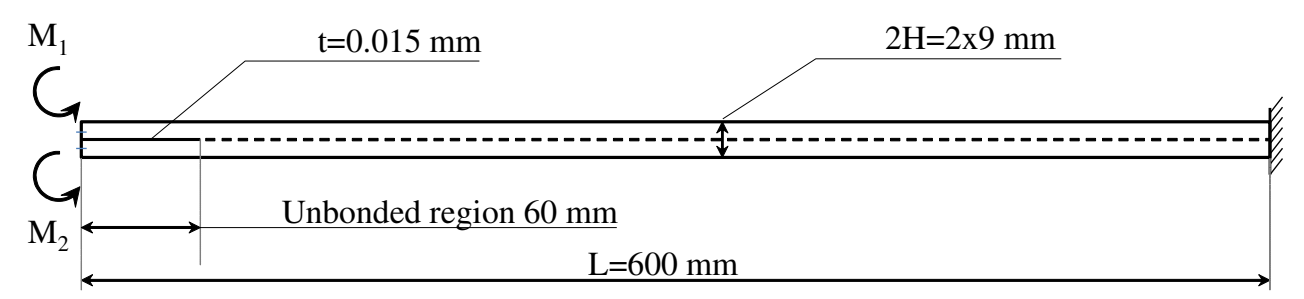

Figure 6: Geometry, constraints and loading of the DCB specimen

During the tests pure bending moments $M_{1}, M_{2}$ were applied at the free ends of the cantilever by a special test fixture using a wire and roller arrangement that allows increasing the magnitude of both $M_{1}, M_{2}$ while keeping fixed their ratio. Several experimental tests were performed, by spanning the ratio $M_{1} / M_{2}$ in the interval $-1 \leq M_{1} / M_{2}<1$.

These tests were numerically reproduced via implicit dynamic FE analyses, which was preferred to quasi static path-following arc-length methods (Alfano and Crisfield, 2003; Riks et al., 1996) in consideration of the improved convergence capabilities of the former. To account for the reported moderately large rotations experimented by the superior and inferior arms of the cantilever, the finitedeformation kinematics option available in the software was activated (Abaqus, 2012). The experimental results were reported by Sørensen and Jacobsen in terms of $J$-integral computed in the assumption of plane strain. For this reason, a two-dimensional plane strain model was used in the FE simulations.

The presented interface formulation was employed to describe the behaviour of the bonded region. In the unbonded region no contact and no friction are considered to account for the presence of the gap created by the slip foil and for 
the positive mode I present in each loading case simulated. The in-plane interface thickness across bonded and unbonded regions is set equal to the reported thickness of the slip foil, of $0.015 \mathrm{~mm}$, applied to prevent adhesion at the left end of the specimens in the experimental test.

For the composite arms, the only available material data reported by Sørensen and Jacobsen were the Young modulus, $E=37 \mathrm{GPa}$, and the Poisson ratio, $\nu=0.3$, which were used by them to postprocess the experimental data based on an isotropic linear elastic model. Accordingly, a simplified linear elastic isotropic model with the same material properties is considered for the composite arms in the simulations. The density of the composite material was taken equal to 2000 $\mathrm{Kgm} / \mathrm{m}^{3}$.

Each arm of the cantilever has been discretized with a uniform structured mesh of 4x230 4-noded linear, fully integrated, plane-strain elements with incompatible modes. On the initially bonded part the interface has been discretized by 207 4-noded interface elements.

As above mentioned, the experimentally recorded moments have been reported in terms of $J$ integral, related to $M_{1}$ and $M_{2}$ in the assumption of plane strain by Sørensen et al. (2006):

$$
J=\left(1-\nu^{2}\right) \frac{21\left(M_{1}^{2}+M_{2}^{2}\right)-6 M_{1} M_{2}}{4 B^{2} H^{3} E}
$$

The quantity $J$ was plotted against the norm $\delta$ of the relative displacement between upper and lower beams at the initial crack tip. These data are reproduced in Figure 7 by point markers for the tests corresponding to the ratios $M_{1} / M_{2}$ reported in the legend.

Calibration of the interface parameters in the bonded region was performed as follows. The fracture energy $G_{c n}=G_{c t}=G_{c}$ was taken as the plateau value of $J$ in the $J$ - $\delta$ curve in pure mode I $\left(M_{1} / M_{2}=-1\right)$. $\sigma_{o n}=\sigma_{o t}$ were estimated based on the pure mode I curves reported by Sørensen and Jacobsen. The slopes of the curves turned out to be rather insensitive to $R_{\eta}$, provided values sufficiently close to unity are taken for such parameter. As a second step, the remaining parameters $\mu$ and $\theta$ were set so as to curve-fit the other mixed-mode responses. The interface parameters resulting from this calibration procedure are collected in Table 2.

\begin{tabular}{|c|c|c|c|c|c|c|}
\hline \hline$\sigma_{\text {on }}[\mathrm{MPa}]$ & $\sigma_{o t}[\mathrm{MPa}]$ & $G_{c n}[\mathrm{~N} / \mathrm{mm}]$ & $G_{c t}[\mathrm{~N} / \mathrm{mm}]$ & $R_{\eta}[-]$ & $\mu[-]$ & $\theta_{\text {int }}[\mathrm{deg}]$. \\
\hline 1.0 & 1.0 & 0.97 & 0.97 & 0.95 & 1.0 & $40^{\circ}$ \\
\hline \hline
\end{tabular}

Table 2: Interface material parameters employed in the DCB-UBM structural examples.

The numerical $J-\delta$ responses, corresponding to the five mode ratios of Figure 7 , are plotted by solid lines in the same figure. As shown by Figure 7 while a 


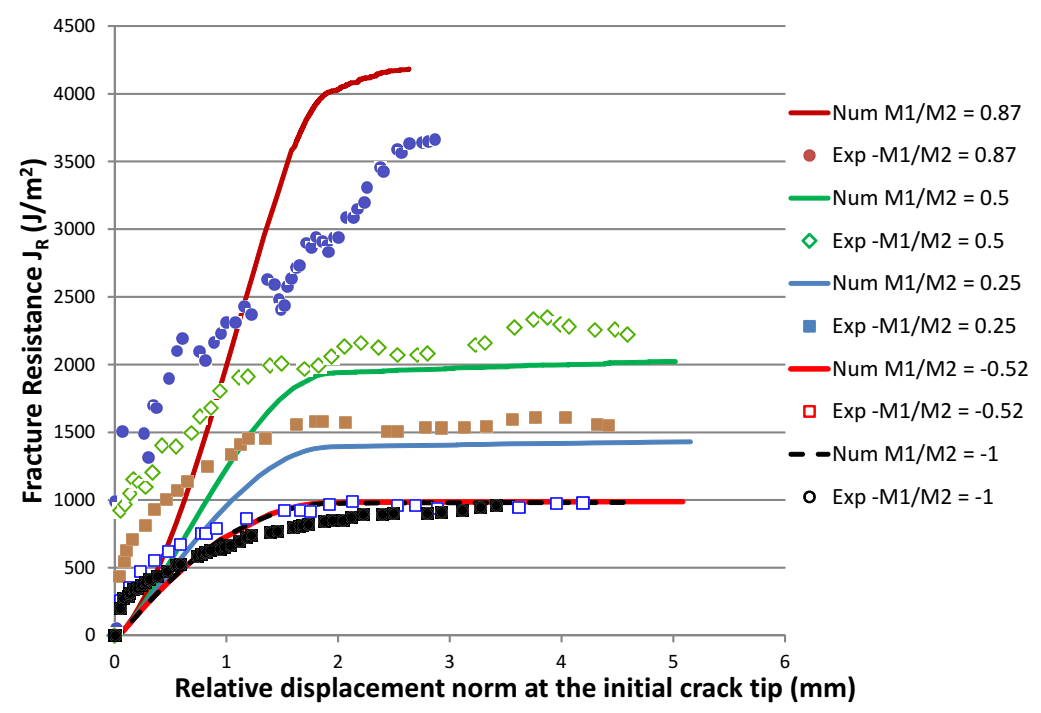

Figure 7: Numerical-experimental comparison of $J$-integral fracture resistance plotted vs. the norm of relative displacement, obtained under different mode mixity ratios

strong quantitative correlation is not expected, since the current interface model does not account for fiber bridging, it can be appreciated that the overall final plateaus for the $J-\delta$ curves, corresponding to steady state crack propagation are well captured. In particular, the numerical model also captures the increase in the steady state values of fracture resistance as the mode II component becomes predominant, i.e., as $M_{1} / M_{2}$ approaches unity. The numerical trend of the $J$ $\delta$ curves observed under mixed mode loading confirms, also at the macroscopic structural level, the capability of the present interface model in predicting the increase in fracture energy under increasing mode II.

\section{Conclusions}

The formulation of a mixed-mode cohesive-zone model (Alfano and Sacco, 2006) accounting for friction interface damage and its recently proposed enhancement to account for friction and interlocking (Serpieri and Alfano, 2011) have been recast within the general theory of thermodynamics with internal variables, with an original derivation of thermodynamic consistency conditions that should be satisfied.

In the presented formulation, the widely used choice of introducing an equivalent relative displacement $s^{e q}$ which suitably combines mode-I and mode-II components is made and the assumption that damage is an increasing function $\hat{\alpha}$ of $s^{e q}$ is made. The model is presented in a very general setting first, and then it 
is specialized to the case of linear elastic behavior for constant damage, linear softening and to specific choices for $s^{e q}$ and $\hat{\alpha}$.

This study shows that use of a single damage variable combined with the choice of having a threshold damage function only depending on the damage variable itself and an equivalent displacement norm requires coincidence of fracture energies in modes I and II for thermodynamic consistency. This result is in agreement with Del Piero and Raous (2010), who observe that, for the construction of a general model of adhesive interfaces with a single damage variable, one single diagonal experiment is sufficient to determine the constitutive behavior.

Furthermore, it is shown that the experimental evidence that the measured fracture energy increases with increasing mode II/mode I ratio can be retrieved by enhancing the model to account for friction and interlocking, based on simplified, yet physically well justified, micro-mechanical assumptions.

Results of microstuctural computations conducted at the single interfacepoint have been reported to show that, within the presented model, the increase of apparent mode II fracture energy emerges as the joint effect of a nonzero friction angle and a nonzero interlocking angle. Interlocking is thus recognized to be a fundamental parameter to be accounted for in order to obtain predictions of different mode I and mode II fracture energies for a single fixed cohesive damage behavior. For concrete-to-concrete interfaces, a possible calibration of the interlocking angle was also shown to retrieve experimentally measured values of mode I and mode II specific fracture energies.

The structural simulations of mixed mode delamination in laminated composite DCB-UBM experiments have shown that the present interface model succeeds in predicting the increase in fracture energy under increasing mode II/mode I ratio with good overall agreement.

The possibility of considering a cohesive relationship in the tangential direction as well as in the normal direction for quasi-brittle materials is a contentious issue, since it is difficult to separate the cohesive-sliding relation from the frictional force between the rough cohesive crack faces. The reported numerical comparisons show that the presented model provides a means for capturing such a separation in a thermodynamically consistent framework employing a microstructural description in which the separate contributions of adhesion, interlocking and friction are indentifiable and are associated with parameters having a specific microstructural meaning.

\section{Acknowledgements}

The first author gratefully acknowledges the financial supports of the Univer-

sity of Sannio. The second author gratefully acknowledges the financial supports 
of the University of Cassino and of the Consorzio RELUIS (Department of Civil Protection).

\section{References}

Abaqus 6.12 Analysis Users Manual, Dassault Systèmes Simulia Corp., Providence, RI, USA, 2012.

Alfano, G. and Crisfield, M. A., 2001. Finite element interface models for the delamination analysis of laminated composites: mechanical and computational issues. International Journal for Numerical Methods in Engineering. 50, 1701-1736.

Alfano, G., Crisfield, M. A., 2003. Solution strategies for the delamination analysis based on a combination of local-control arc-length and line searches. International Journal for Numerical Methods in Engineering, 58(7), 999-1048.

Alfano, G. and Sacco, E., 2006. Combining interface damage and friction in a cohesive-zone model. International Journal for Numerical Methods in Engineering. 68, 542-582.

Allix, O., Corigliano, A., 1996. Modeling and simulation of crack propagation in mixed-modes interlaminar fracture specimens. International Journal of Fracture. 77, 111-140.

Alfano, G., Marfia S., Sacco, E., 2006. A cohesive damage-friction interface model accounting for water pressure on crack propagation. Comput. Meth. Appl. Mech. Engng. 196, 192-209.

Aci Committee, 2005. Building Code Requirements for Structural Concrete (ACI 318-05) and Commentary (ACI 318R-05). American Concrete Institute.

Barpi F., Valente S., 2010. The cohesive frictional crack model applied to the analysis of the dam-foundation joint. Engineering Fracture Mechanics. 77(11), 2182-2191.

Carpinteri, A., Valente, S., Ferrara, G., Melchiorri, G., 1993. Is mode II fracture energy a real material property?. Computers \& Structures. 48(3), 397-413.

Corigliano, A., 1993. Formulation, identification and use of interface models in the numerical analysis of composite delamination. International Journal of Solids \& Structures. 30, 27792811.

Del Piero, G. and Raous, M., 2010. A unified model for adhesive interfaces with damage, viscosity and friction. European Journal of Mechanics A/Solids. 29, 496-507.

Karihaloo B.L., Xiao Q.Z., 2008. Asymptotic fields at the tip of a cohesive crack. International Journal of Fracture, 150, 55-74.

Mi, Y., Crisfield, M.A., Davies, G.A.O., Hellweg, H.B, 1998. Progressive delamination using interface elements. Journal of Composite Materials. 32(14), 1246-1272.

Ragueneau F., La Borderie C., Mazars J., 2000. Damage model for concrete-like materials coupling cracking friction contribution towards structural damping: first uniaxial applications. Mech. Cohes. Frict. Mater., 5, 607-625.

Raous, M., Cangémi, L., Cocu, M., 1999. A consistent model coupling adhesion, friction, and unilateral contact, Comput. Methods Appl. Mech. Engrg., 177, 383-399.

Riks, E., Rankin, C. C., Brogan, F. A., 1996. On the solution of mode jumping phenomena in thin-walled shell structures. Computer methods in applied mechanics and engineering, 136(1), $59-92$.

Sacco, E. and Toti, J., 2010. Interface elements for the analysis of masonry structures. International Journal for Computational Methods in Engineering Science and Mechanics. 11, 354-373.

Serpieri, R., Alfano, G., 2011. Bond-slip analysis via a thermodynamically consistent interface model combining interlocking, damage and friction, International Journal for Numerical Methods in Engineering. 85(2), 164-186.

Simo, J.C., Hughes, T.J.R., 1998. Computational Inelasticity, Springer, Berlin. 
Spada A., Giambanco G., Rizzo P., 2009. Damage and plasticity at the interfaces in composite materials and structures. Comput. Methods Appl. Mech. Engrg., 198(2009) 3884-3901.

Suquet, P., 1982. Plasticité et homogénéisation. Thése de Doctorat d'Etat, Université Paris VI. Sørensen B.F., Jørgensen K., Jacobsen T.K., Østergaard R.C., 2006. DCB-specimen loaded with uneven bending moments. Int. J. Fract., 141, 159-72.

Sørensen, B. F., Jacobsen, T. K., 2009. Characterizing delamination of fibre composites by mixed mode cohesive laws. Composites Science and Technology, 69(3), 445-456. 